\title{
All-Fabric Wearable Electroadhesive Clutch
}

\author{
Vivek Ramachandran, Jun Shintake, and Dario Floreano*
}

\author{
V. Ramachandran, Prof. J. Shintake, Prof. D. Floreano \\ Institute of Microengineering \\ School of Engineering \\ École Polytechnique Fédérale de Lausanne \\ 1015 Lausanne, Switzerland \\ E-mail: dario.floreano@epfl.ch
}

Prof. J. Shintake

Department of Mechanical and Intelligent Systems Engineering

Graduate School of Informatics and Engineering

The University of Electro-Communications

Tokyo 182-8585, Japan

\begin{abstract}
We introduce a lightweight, textile fabricated, haptic device consisting of an electrostatic adhesive clutch, which can constrain body movement when activated at low power $(\sim 1 \mathrm{~mW})$. The clutch electrodes are composite structures, prepared by coating copper-plated polyester fabric with thin films of high- $\kappa$ dielectric ink. When voltage is applied across a pair of overlapping electrodes, the charge separation created between the overlapped surfaces gives rise to adhesive forces that resist tensile loads along the electrode surface. The clutch is arranged in parallel with a sheet of knitted fabric, which exhibits low-stiffness spring-like characteristics, thus decreasing load resistance when the clutch is deactivated. Mechanical tests are carried out to assess the dependency on scaling and loading rate at different voltages. The load-bearing capacity of the device is experimentally shown to sustain a $10 \mathrm{~kg}$ load for a clutch pair with $120 \times 70 \mathrm{~mm}^{2}$ dielectric overlap, when activated at $400 \mathrm{~V}$. We present current-dependent charging and discharging times that can be as low as $15 \mathrm{~ms}$. To exemplify its pertinence in wearable applications, the device
\end{abstract}


is used as an elbow joint constraint, exhibiting its conformability to curvatures and suitability for skin-mounted applications.

\section{Manuscript}

In the field of wearables, the provision of haptic feedback has successfully been shown to supplement the information gathered by the human body's intrinsic sensory mechanisms for applications ranging from rehabilitation to teleoperation $[1,2]$. In fact, with the introduction of new wearable interfaces to control distally-located robots, haptic feedback to the whole body could help users learn to control the robots better than they might with conventional hand-held controllers that have limited physical points of contact with the human skin [3, 4]. One approach is to provide passive haptic feedback i.e., feedback produced by devices that generate mechanical impedance, such as brakes and clutches $[5,6]$. Passive haptic devices are characterised by guides that transmit the impedance which can constrain user movement and prevent them from committing errors. Unlike powered wearable orthotics for neuromuscular ailments, which require rigid components like actuators $[7,8]$, wearable passive haptic devices can be developed exclusively using soft materials. Indeed, in recent years there has been a greater emphasis to incorporate human skin-like elastic materials in wearable systems for improved biomechanical compatibility [9]. In soft matter engineering, the predominant materials used are silicone-based elastomers because they can deform under light mechanical loading and distribute the load to avoid high stress concentrations [10]. However, a key limitation of such elastomers is that they are non-porous. This makes them unsuitable for direct skin-mounted applications because of potential health risks caused by skin occlusion and sweat build-up. One way to address this issue is to use textiles that allow the skin to breathe through their porous texture. Textiles can considerably reduce the negative effects of weight and inflexibility, which are ergonomic factors that affect user-comfort [11]. Not only do these materials have intrinsic mechanical properties that make them compliant and non-restrictive, but they also serve as compatible substrates for stretchable sensors that track pressure change and body flexure [12]. Therefore, such materials would be more suitable for skin interfacing compared to elastomers.

To provide an impedance-based kinesthetic feedback without actuators, which can be transmitted through fabric garments, haptic devices can be fabricated by combining stiffness-tuning technologies with textiles [13, 14]. Previous work has shown that soft-matter devices, which incorporate rigidity tunable materials, retain their inherent softness for safe human interaction, while also being capable of transmitting high loads in their stiff state. In addition to having a rapid rate of response and a large bandwidth of compliance, these devices should ideally be lightweight, inexpensive, safe to use, scalable, and devoid of sizeable external hardware. The more promising variable stiffness technologies have employed either layer or particle jamming $[14,15,16,17,18]$, phase chageable materials $[19,20,21,22]$, or electrostatic adhesion [23]. Among these approaches, jamming technologies suffer from the need for bulky auxiliary equipment to remove air and regulate air pressure. Material phase change, often a thermal process, consumes significant amount of power and takes a long time to respond due to slow heat dissipation. On the other hand, electrostatic adhesion, or electroadhesion, exploits characteristics of capacitors, 
such as low power consumption and short response time, using only lightweight and portable electronic components. Electroadhesion has mostly been used in the field of robotics for gripping, perching and wall-climbing applications $[24,25,26,27,28]$. In most instances, a combination of tacky polymers with moderate conductive and dielectric properties were used to fabricate capacitors [29]. However, the use of intrisically-adhesive polymers could also result in residual stiction and slow disengagement. This problem was addressed recently in work that focussed on the development of an electroadhesive clutch for an ankle prosthesis by using a solid dielectric with a smooth surface [23]. Their device was very effective in controlling the spring engagement of the exoskeleton and returning stored mechanical energy during walking. However, it must be noted that the use of metallised plastic electrodes in their clutches would be unsuitable for textile fabricated devices, arising from materials compatibility issues in the manufacturing process.

In this paper, we present a method to develop wearable haptic devices that are almost entirely fabricated using different types of textiles (Figure 1). These devices incorporate fabric-electrostatic adhesive clutches that provide force feedback through active resistance to tensile loading. The advantage of the method proposed in this paper is the employment of stitching, a common textile manufacturing method. Compared to dry or wet adhesives that create chemical bonds to join surfaces, stitches are more resilient to environmental externalities such as humidity and are more easily mendable in case of damage. In this work, we demonstrate the feasibility of the manufacturing process as well as the material architecture that allows for directional stiffness tuning at low power. Furthermore, we demonstrate the feasibility of the clutch in tensile applications by using it as an elbow joint constraint.

The haptic device is composed of a pair of electroadhesive clutch plates and non-functional knitted and woven fabrics, arranged in the order shown in Figure 1a. Knitted fabrics are stretchable and woven fabrics are inextensible. Each clutch plate consists of a conductive woven textile sheet $(\mathrm{CN}-3190,3 \mathrm{M})$ of pre-determined shape, which is thin-film coated with a high- $\kappa$ dielectric ink (Luxprint 8153, DuPont) and laser-cut knitted and woven fabrics (Figure 1b). The device is comprised of two identical parts, each fabricated by stitching a clutch plate and a piece of woven fabric onto a sheet of knitted fabric, leaving only a portion of the knitted fabric unconstrained (Figure 1c). The two parts are aligned to form a clutch pair, such that the dielectric surfaces of the pair are in planar contact and the unconstrained portions of the knitted fabric are at opposite ends (Figure 1a, see Experimental Section). Finally, we enclosed the entire device in a protective textile covering to prevent plate separation perpendicular to the longitudinal axis (Figure 1d). The fabrication can be modified to incorporate multiple clutch plate pairs that are arranged in parallel to increase the maximum holding force (Figure S5, Supporting Information). The device is designed to have a low-stiffness spring in parallel with the high-holding force clutch. This means that when the clutch is not in operation, the device can comfortably stretch along the human body without exerting a significant restoring force. The assumption here is that the friction between the dielectric surfaces of the clutch plates is negligible. The clutch operates on the same principle governing parallel plate capacitors. When a voltage is applied across the electrodes, charge separation induces Maxwell stress normal to the electrode plane (Figure 1a). This 


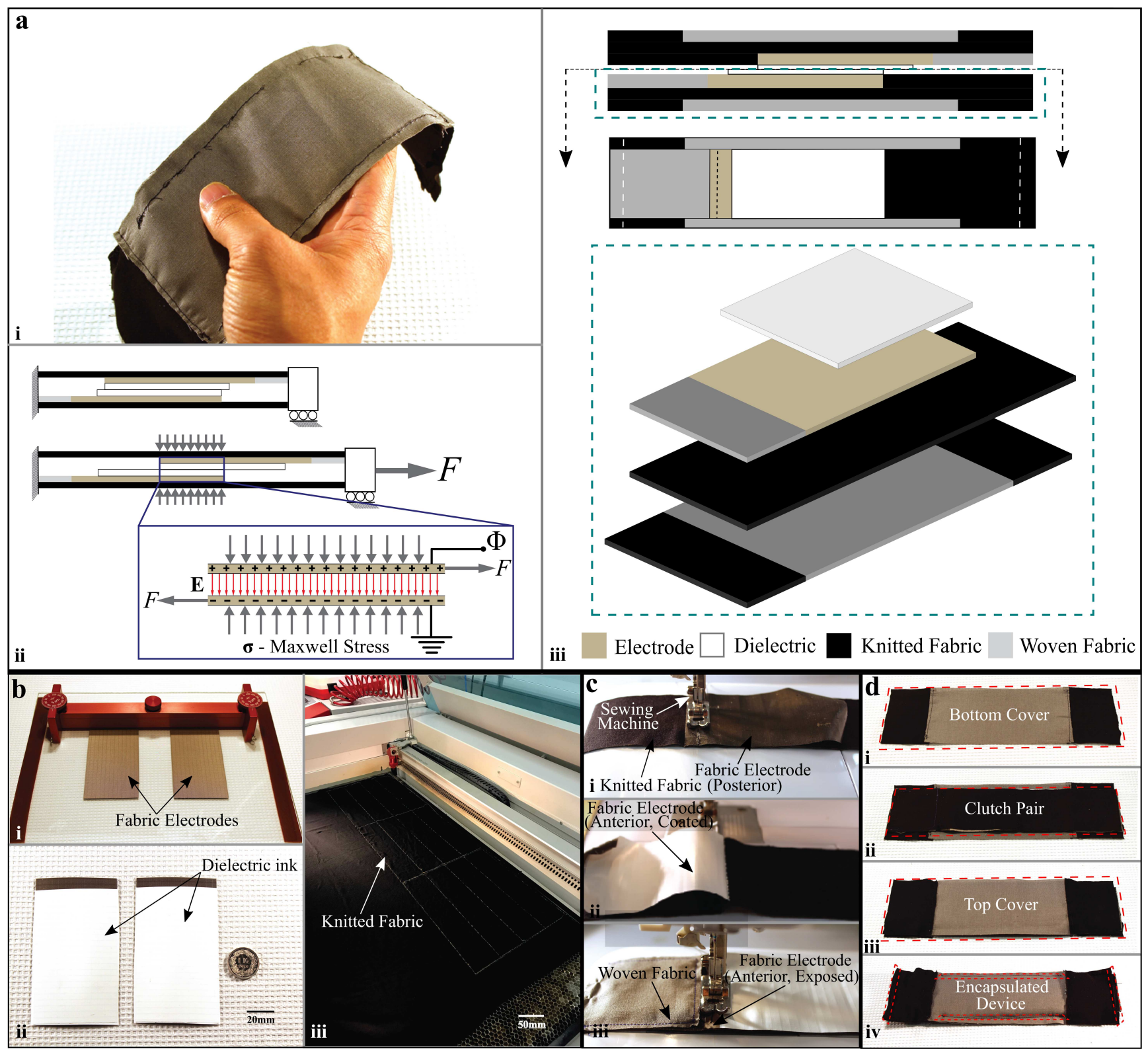

Figure 1: Textile clutch structure, working principle, and fabrication. (a) (i) Encapsulated haptic device with integrated electroadhesive clutch plates. (ii) When the haptic device is stretched longitudinally, the dielectric overlap area reduces. To prevent further stretch, a voltage $\Phi$ is applied across the electrodes that induces Maxwell stress $\sigma$ normal to the electrode surface and increases the frictional force. (iii) Cross-section and exploded view of the clutch pair components. Fabric components are joined by stitches (dashed, white \& black). (b) Component manufacture of the clutch plates and fabric sheets. (i) Two copper plated polyester fabric electrodes are bonded to a glass substrate. (ii) The electrodes are thin-film coated with a dielectric ink. A portion of the electrode is left uncoated to interface with a high voltage power supply. (iii) Knitted fabric is laser cut into rectangular sheets. (c) Assembly of haptic device. (i) The posterior surface of the electrode is stitched onto the knitted substrate along the wider edge of the coated surface. (ii) The electrode is folded to cover the stitched seam and the coated surface is aligned in parallel with the knitted substrate. (iii) The exposed portion of the coated (anterior) electrode face is stitched onto the substrate along with a rectangular laser-cut woven fabric sheet covering the knitted fabric surface between the exposed, anterior electrode portion and the substrate edge. This sample is replicated and aligned such that the coated electrodes are in planar contact and the unconstrained portion of the knitted substrate are longitudinally at opposite ends. (d) Encapsulation in a textile covering. (i) Bottom cover is manufactured by stitching two identical knitted fabric sheets to a single woven fabric sheet. (ii) Clutch pair with overlapping electrodes is placed on top of the bottom cover. (iii) A top cover, identical to the bottom cover, is placed on top of the clutch pair. (iv) The covers are stitched along the longer edges with seams avoiding the clutch pair. The covers and the clutch pair are stitched together along the wider edges to completely encapsulate the device. 
adhesion increases the maximum shear force required to induce slippage when subjected to a tensile load.

For the plate electrode, we chose copper plated polyester fabric tape because it could easily be sewn onto other fabrics. While the tape's acrylic adhesive backing could serve as a weaker, secondary bond to the substrate that could resist shear loading, it could also be delaminated by peeling if replacement was necessary. We selected Luxprint as the dielectric material because it contains Barium Titanate, a ferroelectric crystal commonly used in solid-state ceramic capacitors [30, 31]. The high dielectric constant of Barium Titanate increases the chargecarrying capacity of the capacitor, thereby increasing the adhesive force when a voltage is applied. Luxprint is particularly suitable for the fabrication process as well. In its uncured state, it is a free-flowing, viscous fluid that can be oven-cured into thin films on flat substrates using a thin film applicator. In its cured state, the surface is smooth and thus displays low friction. This physical property is important to ensure high surface conformability, which results in stronger adhesion upon clutch activation, and for providing negligible shear resistance when no voltage is applied [23]. The thin dielectric film bonds well to the flexible fabric without cracking, although the dielectric layer thickness decreases by more than half after the sample is cured in the oven. This phenomenon is due to the fact that the solvent present in the dielectric ink, which gives it its fluidic constitution, evaporates during the curing process and that the ink seeps through the numerous air gaps present in the weaves of the fabric. We performed two sets of experiments in order to characterize the behaviour of the device. In the first set, we measured mechanical characteristics, such as the dependence of the maximum clutching load on the area of dielectric overlap, the effect of number of clutch plate pairs arranged in parallel, and the rate of loading (displacement-controlled and force-controlled). In the second set, we measured the relevant electrical characteristics, such as capacitor charging and discharging time, and the total power consumption.

When a voltage $\Phi$ is applied across the parallel plate capacitor, an electric field is created between the plates and charges begin to flow from one electrode to the other until the potential difference across the capacitor plates equals $\Phi$ (Figure 1a). The capacitor has a capacitance $C=A \kappa \epsilon_{0} / x$, where $A$ is the capacitor planar surface area, $\kappa$ is the dielectric constant, $\epsilon_{0}$ is the dielectric permittivity of free space, and $x$ is the dielectric thickness. The electric field induces Maxwell stress normal to the electrode plane (see Supporting Information). This stress component is responsible for the rapid increase in frictional force along the surface area of the planar electrodes, opposite to the loading direction. For $n$ engaged clutch plate pairs placed parallel to each other, the adhesive force in-plane is given by:

$$
F=\frac{1}{2} \mu \kappa \epsilon_{0} n A\left(\frac{\Phi}{x}\right)^{2}
$$

where $\mu$ is the coefficient of static friction between the surfaces of the dielectric. As shown in Figure 2, we observe the mechanical characteristics of the clutches to be in keeping with expected trends. As Equation (1) suggests, the maximum load that the clutch plates can withstand increases in proportion to the dielectric area of 
overlap and the number of engaged clutch plate pairs (Figure 2a,b). The linear dependency on the dielectric area of overlap is especially relevant when the clutch plates are engaged after the device has been stretched by a certain amount. Here, the dielectric area of overlap reduces from its initial value in the rest configuration and consequently, the maximum holding force capacity also reduces. It must be noted that the force is only dependent on the total surface area of dielectric overlap and not the individual dimensions of the clutch plates. Therefore, the device can be designed with plate dimensions and rest configuration dielectric area of overlap that are capable of handling different magnitudes of load. As predicted by Equation (1), Figure 2c,d also show a quadratic load dependence on the applied voltage. The samples are loaded by defining the rate of loading, either displacement-controlled ( $\mathrm{mm} / \mathrm{s})$ or force-controlled $(\mathrm{N} / \mathrm{s})$. It is worth pointing out that for both sets of tests, the maximum holding force reduces at high rates of loading. The force-controlled tests exhibit a slightly higher variance compared to displacementcontrolled tests at high voltages. Unlike displacement-controlled tests that are open-loop processes where the tests are carried out by specifying a fixed displacement rate, force-controlled tests are closed-loop processes where the loading rate depends on the force measured by the load cell of the commercial tensile tester. Therefore, delays in transmitting the measured value from the load cell to the operating software may have contributed to the aforementioned artefact.

To calculate the charging and discharging times of the capacitor, we used a customized H-bridge (Figure S1, Supporting Information) with a resistor placed in series with the capacitor to determine the capacitor voltage drop by measuring the branch current. When a digital signal is sent from a microcontroller to transistors in one branch of the bridge, the high voltage $\Phi$ is applied across the capacitive load. The voltage drop across the capacitor increases exponentially from zero to the peak value at steady state (Figure 2e). This rise is caused by a corresponding exponential charge build-up on the capacitor electrodes. We calculated the charging time as the time required for the voltage to reach $99.3 \%$ of its peak value. For instance, a single clutch plate pair with a dielectric overlap area of $50 \times 60 \mathrm{~mm}^{2}$ takes $13 \mathrm{~ms}$ to charge when operated at $200 \mathrm{~V}$.

A consequence of capacitance charge build-up is dielectric space charge. Space charge is caused by the induced polarising electric field that aligns the ionic crystals to oppose the applied electric field [32]. When the capacitor plates are shorted, the charges in the capacitor begin to flow in the opposite direction until the voltage across the electrodes is zero. However, the presence of space charge might cause the plates to retain residual stiction that can have significant consequences when operating haptic devices at high frequency. While the space charge for Luxprint is low [23], the discharging time can be reduced even further by driving current in the opposite direction for a brief period of time to quicken space charge removal (see Supporting Information). When operated at $200 \mathrm{~V}$, a clutch pair with a dielectric overlap area of $100 \times 60 \mathrm{~mm}^{2}$ consumes an average power of $0.9 \mathrm{~mW}$ during charging and $1 \mathrm{~mW}$ during discharging. By using the H-bridge to drive current in the opposite direction until the capacitor voltage is driven to zero, the discharge time can be reduced from $15 \mathrm{~ms}$ to $2 \mathrm{~ms}$, while the clutch pair consumes an average power of $2.7 \mathrm{~mW}$ to discharge (Figure 2f). 

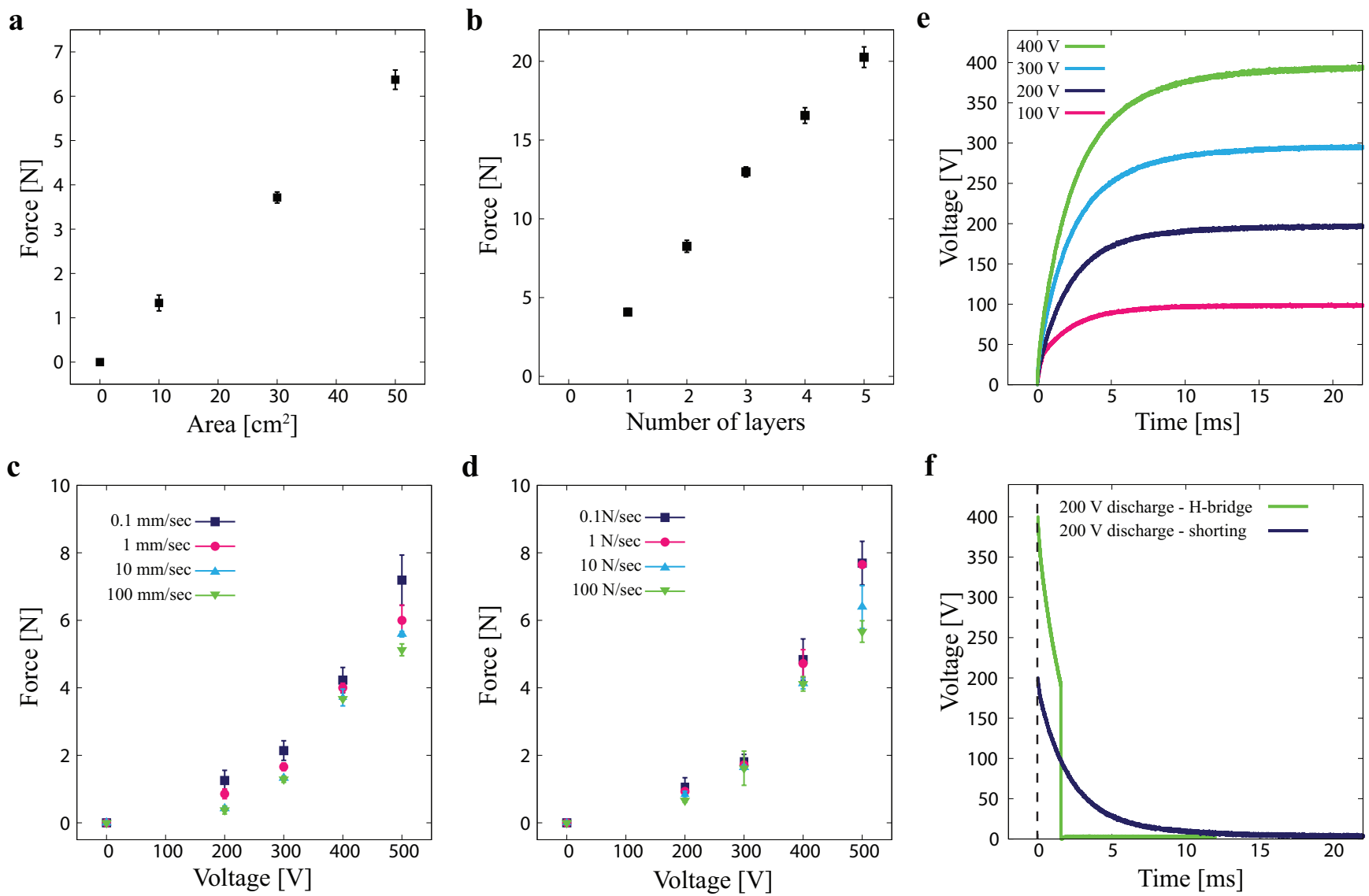

Figure 2: Maximum holding force capacity of the fabricated textile electroadhesive clutch as a response to an applied voltage for varying (a) area of overlap, (b) number of parallel clutch plate pairs, (c) displacement-controlled loading rate, and (d) force-controlled loading rate. Each clutch plate is bonded to a $150 \mathrm{~mm} \times 60 \mathrm{~mm}$ sheet of woven fabric and each clutch pair has an initial dielectric overlap area of $50 \times 60 \mathrm{~mm}^{2}$. (e) Exponential capacitor charging characteristics of a clutch plate pair at different voltages. (f) Clutch plate discharge characteristics observed at $200 \mathrm{~V}$, both by short circuiting and by reversing the voltage polarity using an H-bridge. The discharge characteristics are measured by observing the voltage drop across the measurement resistor placed in series with the capacitor. 


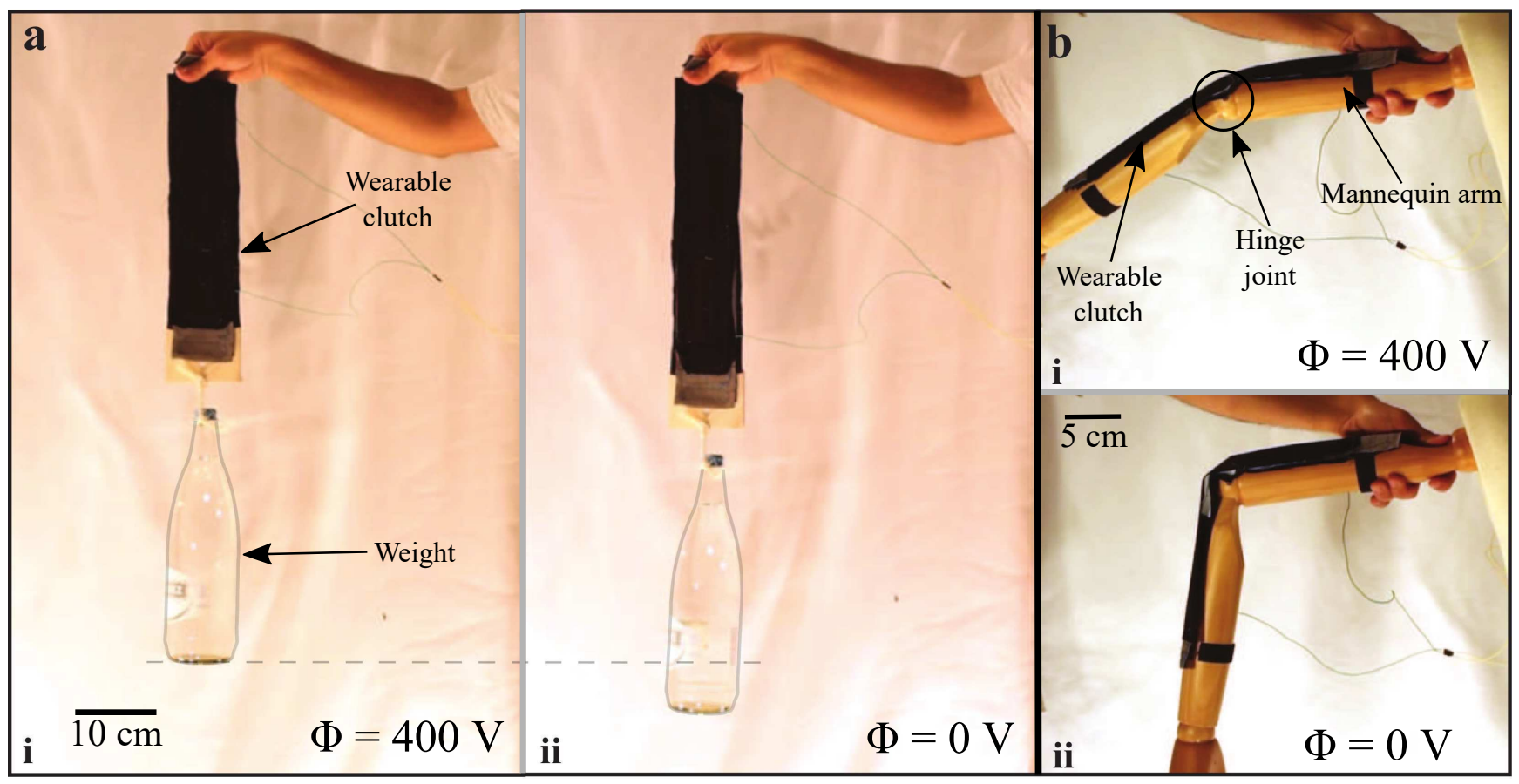

Figure 3: Demonstration of haptic device with single clutch pair having a dielectric overlap area of $120 \times 70 \mathrm{~mm}{ }^{2}$ and engaged at $400 \mathrm{~V}$ for (a) load bearing and (b) skin-mounted applications. (a)-(i) The initially engaged clutch is able to lift a weight of $10 \mathrm{~kg}$. (a)-(ii) Upon disengagement, the knitted fabric stretches like a spring under the influence of the loaded weight. (b)-(i) The engaged clutch is able to constrain the rotation of a mannequin forearm about the elbow hinge joint. (b)-(ii) The disengaged clutch readily allows the forearm rotation without providing comparable resistance to movement

To validate the capabilities of the haptic device, two demonstrations are carried out using a single clutch pair device that weighed $23 \mathrm{~g}$, with rest configuration dielectric overlap area of $120 \times 70 \mathrm{~mm}^{2}$, and operated at $400 \mathrm{~V}$. In the first instance, a 1 litre bottle of water, weighing $10 \mathrm{~kg}$ is hung from one end of the device, with the clutch engaged (Figure 3a and Video S1). The clutch is then disengaged, causing the jersey fabric to stretch and the bottle to lower. For the second demonstration, the device is used to block the movement around a mannequin elbow hinge joint (Figure 3b and Video S2). The arm is positioned such that the shoulder joint is kept locked but the forearm, weighing $8 \mathrm{~kg}$, is free to rotate about the elbow hinge joint. The activated device is capable of supporting the weight of the forearm, thus indicating that it can impart force feedback to block undesirable movement. When the device is not in operation, the forearm rotates under the influence of its own weight and causes a longitudinal stretch of the device.

In summary, we developed a haptic device consisting of an electroadhesive clutch, fabricated using textiles. When the clutch is not activated, the device is allowed to stretch without providing much resistance to loading. Upon activation, a voltage is applied across the electrodes of the clutch which increases the resistance to tensile load. The device is lightweight, flexible, and operates at low power. The best reported work on electrostatic clutches is the one developed for the ankle exoskeleton [23]. It was capable of withstanding a shear stress of 17 $\mathrm{kPa}$ at $120 \mathrm{~V} / \mathrm{mm}$, the device weighed $26 \mathrm{~g}$, and the net power consumption was $0.6 \mathrm{~mW}$ over a period of $30 \mathrm{~ms}$. 
In comparison, the clutch presented here can sustain a maximum shear stress of $3.5 \mathrm{kPa}$ at the same voltage per unit dielectric thickness, weighs $23 \mathrm{~g}$, and consumes $0.9 \mathrm{~mW}$ over a period of $15 \mathrm{~ms}$. The lower shear stress of the fabric clutch can be attributed to the mechanical and electrical properties of the fabric electrode the presence of air gaps between the weaves of the textile reduces the capacitors charge carrying capacity and its surface irregularities lower the effective overlap surface area. However, the use of an all-fabric device greatly facilitates material-level compatibility for integration in textile-based wearable systems and streamlines the manufacturing process for rapid fabrication. In addition, we believe that the mechanical design of a spring and clutch in parallel is an elegant solution for providing passive haptic feedback. Despite requiring high operating voltages, electrostatic adhesion as a technology is safe provided the power-consumption is low i.e., the current required to charge and discharge the circuit is small $(<5 \mathrm{~mA})$.

Given that the holding force increases linearly with the dielectric area of overlap and not the individual dimensions of the clutch plates, it is possible to scale up the device to encompass large parts of the body. For body appendages like fingers where the area to mount the device is small, multiple clutch plate pairs can be stacked to produce sufficient force to provide haptic feedback. Power loss due to leakage through the capacitor is low. Thus, for larger capacitors, power consumption during charging and discharging will largely depend on Joule heating through the series resistance, which limits the current flow through the circuit. Larger capacitors require more time to charge and discharge, but increasing current flow can reduce the reaction time, at the expense of marginally increasing power consumption.

Despite the numerous advantages of using this technology, there are some limitations that need to be addressed. The application of high voltages to Barium Titanate results in a decay of its dielectric properties [33, 34, 35]. The same was indicated through anecdotal observations made over a period of a few months, where repeated clutch charging and discharging led to a deterioration in its holding force. Additionally, Barium Titanate, like other high- $\kappa$ dielectrics, also has a low breakdown strength that limits the operating range of voltages. One way to address this issue is through the addition of conductive fillers that increases the breakdown strength [36, 37]. Alternatively, multiple clutch plates arranged in parallel can be operated at lower voltages instead of engaging a single clutch plate at a high voltage. Another common problem experienced when loading the engaged clutch plate was arc discharge at the electrode edges, where the dielectric coating was sparse. This can be avoided either by coating both surfaces of the electrode with the dielectric ink or by adhering strips of polyimide tape, which has a higher dielectric strength than Luxprint, along the edges of the electrodes. While the risk of sweat induced capacitor short circuiting is highly remote, it can be avoided by using commercial waterproof fabrics for the encapsulation. Future work will focus on implementing the aforementioned methods to address these existing limitations. In addition, we will explore the possibility of developing a whole-body suit with a network of clutches that can provide haptic feedback for wearable robotic applications. 


\section{Experimental Section}

\section{Clutch Fabrication}

The manufacture of each clutch plate pair is a layered process, involving two classes of materials - an electrically conductive electrode and an insulating dielectric. As shown in Figure 1b-i, two $105 \mathrm{~mm} \times 60 \mathrm{~mm}$ sheets of Nickel on Copper-Plated Polyester Fabric tape (CN-3190, 3M), a woven fabric shielding with acrylic adhesive backing, were bonded to a $127 \mu \mathrm{m}$ sheet of Polymethyl methacrylate (PMMA). $50 \mu \mathrm{m}$ layers of dielectric ink (Luxprint 8153, DuPont) were deposited onto the fabric substrates using thin film application (ZUA 2000, Zehntner), leaving only $5 \mathrm{~mm} \times 60 \mathrm{~mm}$ of the fabric exposed. The ink was oven-cured at $140^{\circ} \mathrm{C}$ for 60 minutes. This step was repeated and the resulting solid dielectric layer was $40 \mu \mathrm{m}$ in thickness (Figure 1b-ii). The clutch plates were removed from the PMMA substrate.

\section{Haptic Device Fabrication}

Rectangular sheets of knitted fabric $(280 \mathrm{~mm} \times 60 \mathrm{~mm})$ and woven fabric $(60 \mathrm{~mm} \times 60 \mathrm{~mm})$ were laser cut, as shown in Figure 1b-iii, using a commercial $\mathrm{CO}_{2}$ laser cutter (Speedy 400, Trotec). Using one sheet of knitted fabric as a substrate, the posterior, uncoated surface of one fabric clutch plate was stitched onto it using a commercial sewing machine (H Class E20, Husqvarna Viking), $150 \mathrm{~mm}$ from the substrate edge (Figure 1c-i). Then, the plate was folded backwards to cover the seam (Figure 1c-ii), such that the coated surface of the plate was aligned with the longer edge of the knitted fabric substrate. The exposed portion of the coated surface was stitched onto the substrate (Figure 1c-iii), along with one sheet of the laser cut woven fabric, thereby covering the portion of the knitted fabric surface between the covered seam of the electrode and the substrate edge. As a consequence, only $130 \mathrm{~mm} \times 60 \mathrm{~mm}$ of the knitted substrate remained unconstrained. The sample was replicated and aligned, such that the coated electrodes were in planar contact and the unconstrained portions of the jersey fabric sheets were at opposite ends. The clutch pair was encapsulated by embedding it between two layers of textile covering sheets - each layer consisted of a woven fabric sheet $(180 \mathrm{~mm} \times 80 \mathrm{~mm})$ stitched to two identical jersey fabric sheets $(60$ $\mathrm{mm} \times 80 \mathrm{~mm}$ ) along its wider edges (Figure 1d). The covering layers were stitched together along the longer edges without the clutch pair. The two layers along with the clutch pair were stitched together along the wider edges. The total device weighed $23 \mathrm{~g}$. An illustrative description of the device fabrication is provided in the Video S3 and the Supporting Information.

\section{Characterisation of Mechanical Properties}

Each clutch plate was bonded onto $150 \mathrm{~mm} \times 60 \mathrm{~mm}$ rectangular sheets of laser cut woven fabric, by iron pressing the woven cloth onto the acrylic adhesive backing of the fabric tape. The melted adhesive created a strong bond between the plate and the fabric. Force-displacement tests of the clutch pairs were conducted with a materials testing machine (Instron 5965). The plates of the pair were fixed to the vices of the tensile tester, such that the dielectric surfaces were in planar contact with each other. To test the dependence of maximum holding force on applied voltage, the vertical separation between the vices was adjusted to ensure an overlap area of $50 \times 60 \mathrm{~mm}^{2}$. 
Prior to commencing each test, voltage was applied to the clutch electrodes using a high voltage supply (PS 350, Stanford Research Systems). The clutch pairs were engaged at 200 V, 300 V, 400 V, and 500 V and loaded under force-controlled conditions $(0.1,1,10,100 \mathrm{~N} / \mathrm{s})$ and displacement-controlled conditions $(0.1,1,10,100 \mathrm{~mm} / \mathrm{s})$. To test the dependence of maximum holding force on area of overlap, tests were carried out for the following areas: $10 \mathrm{~cm}^{2}, 30 \mathrm{~cm}^{2}, 50 \mathrm{~cm}^{2}$, while the plates were engaged at $400 \mathrm{~V}$ and the vices were loaded at $10 \mathrm{~mm} / \mathrm{s}$. To test the effect of number of clutch plate pairs operating in parallel, experiments were carried out using $1,2,3,4$, and 5 pairs of clutch plates. Each characterisation study was carried out for 5 trials.

\section{Dynamic Electrical Characterisation Tests}

To calculate the charging and discharging times for the capacitor, a customized H-bridge with four identical transistors (1NK60Z, STMicroelectronics) was employed (Figure S2, Supporting Information). The clutch plates are interfaced with the H-bridge by soldering wires to the textile electrodes. The H-bridge also consisted of a 2 $\mathrm{M} \Omega$ resistor placed in series with the capacitive load, to measure the current flow through the individual branches and calculate the voltage drop across the capacitor. We denote the transistor of the H-bridge that were closer to the ground potential as LTs and the transistors closer to the high voltage supply as HTs. Initially, LTs were closed and HTs were kept open. When a $5 \mathrm{~V}$ digital signal was sent from the microcontroller (Arduino Nano, Arduino LLC) to close the HT of one branch of the bridge and open the LT of the second branch, a high voltage (100 V, $200 \mathrm{~V}, 300 \mathrm{~V}$, or $400 \mathrm{~V}$ ) was applied across the first branch. The current flow was measured and stored using a digital oscilloscope (HMO2024, Rohde \& Schwarz). The time required for the current to decay to $0.7 \%$ of its peak value was calculated as the charging time. Capacitor discharging was instigated by opening the HT of the first branch and closing the LT of the second branch, thus shorting the circuit and reverting to the initial condition. The current flow, voltage drop, and discharge time were measured in the same manner as the charging phase, using the oscilloscope across the measurement resistor. Discharge time was shortened even further using the H-bridge by two steps that were performed simultaneously. One, the HT of the first branch was opened and the LT of the second branch was closed and two, the HT of the second branch was closed and the LT of the first branch was opened. This resulted in the capacitor current and voltage drop peaking in the second branch to twice the peak value recorded in the first branch, before undergoing exponential decay. Based on experimental data collected for discharge through branch short circuiting, the time needed to reduce the voltage to half was determined. The second branch was kept closed for that exact period of time, after which the HT of the second branch was opened and the LT of the first branch was closed, returning to the initial condition. The capacitor discharge time was measured as the sum total of the time required for the current to drop to $0.7 \%$ of the peak value starting from when the first branch was opened.

\section{Demonstration details}

To demonstrate the load bearing capacity of a single clutch pair device, a 1 litre bottle of water weighing 10 $\mathrm{kg}$ was attached to a plank of wood using a bowline knot (Figure 3a). A $75 \mathrm{~mm} \times 40 \mathrm{~mm}$ rectangular piece of 
Velcro with adhesive backing was attached to one of the surfaces of the plank. The mating Velcro portion of the same dimensions was attached to one end of the haptic device. Initially, the clutch was engaged at $400 \mathrm{~V}$, with a dielectric area overlap of $120 \times 70 \mathrm{~mm}^{2}$. The clutch along with the bottle was lifted to a height of $1 \mathrm{~m}$ above the ground and held. Keeping the top end of the device fixed, the clutch was disengaged and the knitted fabric extended downwards under the influence of the attached weight. To show the device's ability to constrain human body movement, a mannequin arm with a hinge elbow joint was used. Velcro strips were adhered along the 18 $\mathrm{mm}$ circumferences of the forearm and upper arm. With respect to the hinge joint, the strips were $12 \mathrm{~cm}$ along the forearm and the upper arm each and the clutch was attached along the arm's posterior side. When the clutch was disengaged, the $8 \mathrm{~kg}$ forearm rotated about the elbow hinge joint freely, allowing the knitted fabric to stretch. Upon engagement, the rotation was constrained and the forearm was held fixed. When the clutch was disengaged, the forearm was able to rotate freely again.

\section{Supporting Information}

Supporting Information is available from the Wiley Online Library or from the author.

\section{Acknowledgements}

The authors would like to thank Stuart Diller for his insightful comments pertaining to the manufacture of electroadhesive clutches and Olexandr Gudozhnik for fabricating the customised H-bridge used for clutch operation. The authors would also like to thank members of the Laboratory of Intelligent Systems at EPFL for their constructive feedback that strengthened the quality of the manuscript. This work was supported by the Swiss National Science Foundation through the Swiss National Centre of Competence in Research (NCCR) Robotics, by the Japanese Ministry of Education, Culture, Sports, Science and Technology (MEXT) through the Leading Initiative for Excellent Young Researchers (LEADER), and through the project RoboComm++.

\section{Conflict of Interest}

The authors declare no conflict of interest.

\section{Keywords}

Wearable robotics, Soft robotics, Stiffness Tuning, Haptics, Teleoperation.

\section{Corresponding Author:}

Prof. Dario Floreano

EPFL STI IMT LIS, MED 11026 (Batiment MED), Station 9, CH-1015 Lausanne.

dario.floreano@epfl.ch; +41216935230

\section{References}

[1] David A Abbink, Mark Mulder, and Erwin R Boer. Haptic shared control: smoothly shifting control authority? Cognition, Technology \& Work, 14(1):19-28, 2012. 
[2] Abhishek Gupta and Marcia K O'Malley. Design of a haptic arm exoskeleton for training and rehabilitation. IEEE/ASME Transactions on mechatronics, 11(3):280-289, 2006.

[3] Carine Rognon, Stefano Mintchev, Fabio Dell'Agnola, Alexandre Cherpillod, David Atienza, and Dario Floreano. Flyjacket: An upper body soft exoskeleton for immersive drone control. IEEE Robotics and Automation Letters, 3(3):2362-2369, 2018.

[4] Joshua M Peschel and Robin R Murphy. Human interfaces in micro and small unmanned aerial systems. In Handbook of Unmanned Aerial Vehicles, pages 2389-2403. Springer, 2015.

[5] Louis B Rosenberg. Virtual fixtures: Perceptual tools for telerobotic manipulation. In Virtual Reality Annual International Symposium, 1993., 1993 IEEE, pages 76-82. IEEE, 1993.

[6] Vincent Hayward, Oliver R Astley, Manuel Cruz-Hernandez, Danny Grant, and Gabriel Robles-De-La-Torre. Haptic interfaces and devices. Sensor Review, 24(1):16-29, 2004.

[7] Aaron M Dollar and Hugh Herr. Lower extremity exoskeletons and active orthoses: challenges and state-ofthe-art. IEEE Transactions on robotics, 24(1):144-158, 2008.

[8] Michael Goldfarb, Brian E Lawson, and Amanda H Shultz. Realizing the promise of robotic leg prostheses. Science translational medicine, 5(210):210ps15-210ps15, 2013.

[9] Michael Wehner, Brendan Quinlivan, Patrick M Aubin, Ernesto Martinez-Villalpando, Michael Baumann, Leia Stirling, Kenneth Holt, Robert Wood, and Conor Walsh. A lightweight soft exosuit for gait assistance. In Robotics and Automation (ICRA), 2013 IEEE International Conference on, pages 3362-3369. IEEE, 2013.

[10] Carmel Majidi. Soft robotics: a perspectivecurrent trends and prospects for the future. Soft Robotics, 1(1):511, 2014.

[11] Rebeccah Pailes-Friedman. Electronics and fabrics: The development of garment-based wearables. Advanced Materials Technologies, page 1700307, 2018.

[12] Asli Atalay, Vanessa Sanchez, Ozgur Atalay, Daniel M Vogt, Florian Haufe, Robert J Wood, and Conor J Walsh. Batch fabrication of customizable silicone-textile composite capacitive strain sensors for human motion tracking. Advanced Materials Technologies, 2(9), 2017.

[13] Mariangela Manti, Vito Cacucciolo, and Matteo Cianchetti. Stiffening in soft robotics: A review of the state of the art. IEEE Robotics \&3 Automation Magazine, 23(3):93-106, 2016.

[14] A Tonazzini, J Shintake, C Rognon, V Ramachandran, S Mintchev, and D Floreano. Variable stiffness strip with strain sensing for wearable robotics. In 2018 IEEE International Conference on Soft Robotics (RoboSoft), pages 485-490. IEEE, 2018. 
[15] Inrak Choi, Nick Corson, Lizzie Peiros, Elliot W Hawkes, Sean Keller, and Sean Follmer. A soft, controllable, high force density linear brake utilizing layer jamming. IEEE Robotics and Automation Letters, 3(1):450-457, 2018.

[16] Yong-Jae Kim, Shanbao Cheng, Sangbae Kim, and Karl Iagnemma. A novel layer jamming mechanism with tunable stiffness capability for minimally invasive surgery. IEEE Transactions on Robotics, 29(4):1031-1042, 2013.

[17] Eric Brown, Nicholas Rodenberg, John Amend, Annan Mozeika, Erik Steltz, Mitchell R Zakin, Hod Lipson, and Heinrich M Jaeger. Universal robotic gripper based on the jamming of granular material. Proceedings of the National Academy of Sciences, 107(44):18809-18814, 2010.

[18] Sean Follmer, Daniel Leithinger, Alex Olwal, Nadia Cheng, and Hiroshi Ishii. Jamming user interfaces: programmable particle stiffness and sensing for malleable and shape-changing devices. In Proceedings of the 25th annual ACM symposium on User interface software and technology, pages 519-528. ACM, 2012.

[19] Alice Tonazzini, Stefano Mintchev, Bryan Schubert, Barbara Mazzolai, Jun Shintake, and Dario Floreano. Variable stiffness fiber with self-healing capability. Advanced Materials, 28(46):10142-10148, 2016.

[20] Steven Rich, Sung-Hwan Jang, Yong-Lae Park, and Carmel Majidi. Liquid metal-conductive thermoplastic elastomer integration for low-voltage stiffness tuning. Advanced Materials Technologies, 2(12), 2017.

[21] Bryan E Schubert and Dario Floreano. Variable stiffness material based on rigid low-melting-point-alloy microstructures embedded in soft poly (dimethylsiloxane)(pdms). Rsc Advances, 3(46):24671-24679, 2013.

[22] Wanliang Shan, Tong Lu, and Carmel Majidi. Soft-matter composites with electrically tunable elastic rigidity. Smart Materials and Structures, 22(8):085005, 2013.

[23] Stuart Diller, Carmel Majidi, and Steven H Collins. A lightweight, low-power electroadhesive clutch and spring for exoskeleton actuation. In Robotics and Automation (ICRA), 2016 IEEE International Conference on, pages 682-689. IEEE, 2016.

[24] Jun Shintake, Samuel Rosset, Bryan Schubert, Dario Floreano, and Herbert Shea. Versatile soft grippers with intrinsic electroadhesion based on multifunctional polymer actuators. Advanced Materials, 28(2):231-238, 2016.

[25] MA Graule, P Chirarattananon, SB Fuller, NT Jafferis, KY Ma, M Spenko, R Kornbluh, and RJ Wood. Perching and takeoff of a robotic insect on overhangs using switchable electrostatic adhesion. Science, 352(6288):978$982,2016$. 
[26] Harsha Prahlad, Ron Pelrine, Scott Stanford, John Marlow, and Roy Kornbluh. Electroadhesive robotswall climbing robots enabled by a novel, robust, and electrically controllable adhesion technology. In Robotics and Automation, 2008. ICRA 2008. IEEE International Conference on, pages 3028-3033. IEEE, 2008.

[27] GJ Monkman, PM Taylor, and GJ Farnworth. Principles of electroadhesion in clothing robotics. International Journal of Clothing Science and Technology, 1(3):14-20, 1989.

[28] GJ Monkman. Dielectrophoretic enhancement of electrorheological robotic actuators. Mechatronics, 3(3):305$313,1993$.

[29] Federico Carpi, Danilo De Rossi, Roy Kornbluh, Ronald Edward Pelrine, and Peter Sommer-Larsen. Dielectric elastomers as electromechanical transducers: Fundamentals, materials, devices, models and applications of an emerging electroactive polymer technology. Elsevier, 2011.

[30] Shepard Roberts. Dielectric and piezoelectric properties of barium titanate. Physical Review, $71(12): 890,1947$.

[31] Ali Kilic, Eunkyoung Shim, Bong Yeol Yeom, and Behnam Pourdeyhimi. Improving electret properties of pp filaments with barium titanate. Journal of Electrostatics, 71(1):41-47, 2013.

[32] Gorur Govinda Raju. Dielectrics in electric fields. CRC press, 2016.

[33] GA Schneider and V Heyer. Influence of the electric field on vickers indentation crack growth in batio3. Journal of the European Ceramic Society, 19(6-7):1299-1306, 1999.

[34] KnW Plessner. Ageing of the dielectric properties of barium titanate ceramics. Proceedings of the Physical Society. Section B, 69(12):1261, 1956.

[35] Rainer Waser, Tudor Baiatu, and Karl-Heinz Härdtl. dc electrical degradation of perovskite-type titanates: I, ceramics. Journal of the american ceramic society, 73(6):1645-1653, 1990.

[36] Xiaoliang Dou, Xiaolin Liu, Yong Zhang, Huan Feng, Jian-Feng Chen, and Song Du. Improved dielectric strength of barium titanate-polyvinylidene fluoride nanocomposite. Applied physics letters, 95(13):132904, 2009 .

[37] Enis Tuncer, Isidor Sauers, D Randy James, Alvin R Ellis, M Parans Paranthaman, Amit Goyal, and Karren L More. Enhancement of dielectric strength in nanocomposites. Nanotechnology, 18(32):325704, 2007. 